\title{
ON GOMMUTATION SEMIGROUPS OF A GROUP
}

\author{
N. D. GUPTA \\ (Received 28 June 1964, revised 3 March 1965)
}

\section{Introduction}

Let $G$ be a group. With each element $a$ in $G$ we associate the mappings $\rho(a)$ and $\lambda(a)$ of $G$ into itself defined as follows,

$$
g \rho(a)=[g, a] \text { for all } g \in G
$$

$$
g \lambda(a)=[a, g] \text { for all } g \in G \text {. }
$$

The product of mappings is defined as usual. Let $P(G)$ and $\Lambda(G)$ denote respectively the semigroups generated by the set of all $\rho$ 's and $\lambda$ 's. These semigroups will be called the commutation semigroups of $G$.

One naturally raises the following two questions:

(i) Are the commutation semigroups of a group isomorphic?

(ii) If not, how are these two semigroups related to each other?

The answer to both the questions depends upon the group $G$. For instance it can be observed easily that if $G$ is the symmetric group on 3 letters, then $|P(G)|=6$ and $|\Lambda(G)|=9$; and also in this case $P(G)$ is properly contained in $A(G)$.

The purpose of this paper is to give a complete answer to question (i) for dihedral groups and for nilpotent groups which are not of class 4 . The paper is in two parts, dealing with the commutation semigroups of dihedral groups and of nilpotent groups, respectively. For dihedral groups we give criteria for isomorphism of $P(G)$ and $\Lambda(G)$. For nilpotent groups we construct an example of a group of class 5 and prove that for nilpotent groups of class $\geqq 5$, the commutation semigroups are not in general isomorphic. Also we prove that these semigroups are always isomorphic for groups of class 2 and 3. For groups of class 4 we prove, however, that the two semigroups are of the same cardinality.

I thank my supervisor Professor B. H. Neumann F.A.A., F.R.S. for suggesting the study of commutation semigroups and for his general guidance. I also thank the referee for his many useful suggestions which in particular have considerably simplified the proof of theorem 1. 


\section{Notations and definitions}

For any two elements $a$ and $b$ of a group $G$, we write 2.1

$$
a^{b}=b^{-1} a b \text {. }
$$

The commutator of $a$ and $b$ is defined as,

$$
[a, b]=a^{-1} b^{-1} a b ;
$$

and for $n>2$, the left normed commutator of weight $n$ is defined inductively as

$$
\left[a_{1}, a_{2}, \cdots, a_{n}\right]=\left[\left[a_{1}, a_{2}, \cdots, a_{n-1}\right], a_{n}\right] .
$$

The following commutator identities are standard and are used repeatedly without reference,

$$
\begin{aligned}
2.4 & {[a, b] } & =\left[b^{-1}, a\right]^{b}=\left[b, a^{-1}\right]^{a} \\
2.5 & {[a, b c] } & =[a, c][a, b]^{c} \\
2.6 & {[a b, c] } & =[a, c]^{b}[b, c] .
\end{aligned}
$$

If $A$ and $B$ are subgroups of a group $G$, then $[A, B]$ is the subgroup of $G$ generated by all the commutators of the form $[a, b]$ where $a \in A$ and $b \in B$. In particular $[G, G]$ is the commutator subgroup of $G$ and is denoted by $G^{\prime}$. If $G^{\prime}$ is abelian, then $G$ is called metabelian. If $G$ is metabelian and if $[a, b]=1$ for $a, b \in G$, then it can be verified that for any $g \in G$,

$$
[g, a, b]=[g, b, a] .
$$

For a given group $G$, the commutation semigroups $P(G)$ and $A(G)$ may also be written as $P$ and $\Lambda$ respectively. Both $P(G)$ and $\Lambda(G)$ possess zero elements, namely $\rho(1)$ and $\lambda(1)$, respectively; and $\rho(1)=\lambda(1)$. We denote the zero element of $P$ and $\Lambda$ by 0 . For all elements $z$ in the centre of $G$, we have $\rho(z)=\lambda(z)=0$.

An element $\alpha \in P(G)(\Lambda(G))$ is called prime if it cannot be expressed as a product of two or more elements of $P(G)(\Lambda(G))$.

\section{PART I}

\section{Commutation semigroups of dihedral groups}

Let $n=2^{r} m$ be a positive integer where $m$ is odd and $r \geqq 0$. Consider the dihedral group $G$ of order $2 n$ given as:

$$
G=\operatorname{gp}\left\{a, b \mid a^{2}=1=b^{n}, a b a=b^{-1}\right\} .
$$


Let $N$ denote the set of residue classes $(\bmod n)$. For each pair of elements $i, j \in N$, define a mapping $\mu(i, j)$ of $G$ into itself as,

$$
b^{k} \mu(i, j)=b^{k i}, a b^{k} \mu(i, j)=b^{k i+j} .
$$

Then it is easy to see that

$$
\mu(i, j)=\mu\left(i^{\prime}, j^{\prime}\right) \text { if and only if } i=i^{\prime}, j=j^{\prime}
$$

and

$$
\mu(i, j) \mu\left(i^{\prime}, j^{\prime}\right)=\mu\left(i i^{\prime}, j i^{\prime}\right) .
$$

Thus the set of all $\mu(i, j)$ form a semigroup $S$ of order $n^{2}$. Further it can be directly verified that

$$
\rho\left(b^{-j}\right)=\mu(0,-2 j), \rho\left(a b^{-j}\right)=\mu(-2,-2 j)
$$

and

$$
\lambda\left(b^{-j}\right)=\mu(0,2 j), \lambda\left(a b^{-j}\right)=\mu(2,2 j) .
$$

Thus by (2), (3), (4), (5) it follows that $P(G)$ is the subsemigroup of $S$ consisting of all elements of the form $\mu(0,-2 j)$ and $\mu\left((-2)^{l},(-2)^{l} j\right)$; and $\Lambda(G)$ is the subsemigroup of $S$ consisting of all elements of the form $\mu(0,2 j)$ and $\mu\left(2^{l}, 2^{l} j\right)$.

If $u, v$ are relatively prime integers, let $\operatorname{ind}_{u} v$ denote the least positive integer $t$ such that $v^{t} \equiv 1(\bmod u)$. Then we prove the following theorem:

Theorem 1. $P(G) \cong A(G)$ if and only if ind $2 \equiv 0(\bmod 4)$ for every prime divisor $p$ of $m$.

PROof. Let $\operatorname{ind}_{p} 2 \equiv 0(\bmod 4)$ for each prime divisor $p$ of $m$. Let $0 \neq k \in N$ and let $d=(k, m)$ be the greatest common divisor of $k$ and $m$. Let $d<m$. The residue classes $(\bmod m / d)$ which are prime to $m / d$ form a multiplicative group $H$ of order $\varphi(m / d)$, the Euler's function of $m / d$. Let $C_{1}$ and $C_{2}$ denote the cyclic subgroups of $H$ generated by the residue classes of 2 and -2 respectively. By hypothesis both $C_{1}$ and $C_{2}$ are of the same even order. Then clearly either $C_{1}=C_{2}$ or $C_{1} C_{2}=C_{1} \cup-4 C_{1}=$ $C_{2} \cup-4 C_{2}$ and in the latter case if $\left\{m_{1}(=1), m_{2}, \cdots, m_{3}\right\}$ is a set of coset representatives of $C_{1} C_{2}$ in $H$, then $\left\{m_{1}, m_{2}, \ldots, m_{1},-4 m_{1}\right.$ $\left.-4 m_{2}, \ldots,-4 m_{8}\right\}$ is a set of common coset representatives of $C_{1}$ and $C_{2}$ in $H$. Let $M$ denote the set of common coset representatives of $C_{1}$ and $C_{2}$ in $H$. Then,

$$
k=(-2)^{t_{1}} l_{1}=2^{t_{2}} l_{2},
$$

for some $l_{1}, l_{2} \in M$, where $l_{1}, l_{2}, t_{1}, t_{2}$ are uniquely determined.

Now, for each $k \in N$ we define integers $\alpha(k), \beta(k) \in N$ as follows: 


$$
\alpha(0)=\beta(0)=0 \text {; }
$$

$\alpha(k)=\beta(k)=(-1)^{t} k$ if $d=m$, where $k \neq 0$ and $t$ is the largest power of 2 dividing $k ; \alpha(k)=(-1)^{t_{1}} k, \beta(k)=(-1)^{t_{2}} k$ if $d<m$, where $k \neq 0$ and $t_{1}, t_{2}$ are given by $(6)$. The following properties can be easily verified:

$$
\begin{aligned}
& k=k \text { if and only if } \alpha(k)=\alpha\left(k^{\prime}\right) \text { and } \beta(k)=\beta\left(k^{\prime}\right) . \\
& \alpha(1)=\beta(1)=1 \\
& \alpha\left((-2)^{l} k\right)=2^{l} \alpha(k), \beta\left(2^{l} k\right)=(-2)^{l} \beta(k) \\
& \alpha(\beta(k))=\beta(\alpha(k))=k .
\end{aligned}
$$

We now define the mappings $\eta_{1}$ and $\eta_{2}$ of $P(G)$ into $\Lambda(G)$ and $\Lambda(G)$ into $P(G)$ respectively as follows:

$$
\begin{aligned}
& \mu(u, v) \eta_{1}=\mu(\alpha(u), \alpha(v)) \\
& \mu(u, v) \eta_{2}=\mu(\beta(u), \beta(v)) .
\end{aligned}
$$

By (8), (9), (10) both $\eta_{1}$ and $\eta_{2}$ are well defined mappings, and by (11), $\eta_{1}$ and $\eta_{2}$ are inverses of one another. A product in $P(G)$ has the form $\mu(u, v) \mu\left(u^{\prime}, v^{\prime}\right)=\mu\left(u u^{\prime}, v u^{\prime}\right)$ with $u^{\prime}=0$ or $(-2)^{l}$. Then by (9) and (10) $\eta_{1}$ is a homomorphism. Hence $P(G) \cong \Lambda(G)$.

Conversely, let $P(G) \cong \Lambda(G)$. If $m=1$, there is nothing to prove. Let $m>1$ and let $T$ denote the subset of $N$ with elements of the form $2 j$.

Let $\eta$ be an isomorphism of $P(G)$ onto $A(G)$. Since $\mu(0,0)$ is the zero element of $P(G)$ and $\Lambda(G), \mu(0,0) \eta=\mu(0,0)$. Further, since $m>1$, $P(G) \mu(u, v)=\mu(0,0)$ is satisfied precisely when $u=0$. Thus $\mu(0, v) \eta=$ $\mu(0, \theta(v))$ for some permutation $\theta$ of $T$. Suppose $\mu(-2,0) \eta=\mu(c, e)$; then $c=2^{i}$ for some $l$. Since $\left(\mu(0, v) \mu^{t}(-2,0)\right) \eta=\mu(0, \theta(v)) \mu^{t}(c, e)=$ $\mu\left(0, c^{\imath} \theta(v)\right)$; we have,

$$
\mu\left(0,(-2)^{t} v\right) \eta=\mu\left(0, c^{t} \theta(v)\right) .
$$

In particular, since $\theta$ is $1-1$, for all $t=1,2, \cdots$,

$$
\left((-2)^{t}-1\right) x=0 \text { and } \quad\left(c^{t}-1\right) x=0
$$

have the same number of solutions $x \in T$. Now $\mu(-2,0)$ and $\mu(c, e)$ both generate cyclic sub-semigroups of $P(G)$ and $\Lambda(G)$ respectively and by the isomorphism condition it is easy to see that $c$ and 2 are powers of one another, so that

$$
\left((-2)^{t}-1\right) x=0 \quad \text { and } \quad\left(2^{t}-1\right) x=0
$$

have the same number of solutions $x \in T$. Let $d_{1}=\left((-2)^{t}-1, n\right)$ and $d_{2}=\left(2^{t}-1, n\right)$. Then the number of solutions are $\varepsilon d_{1}$ and $\varepsilon d_{2}$ 
respectively where $\varepsilon=\frac{1}{2}$ or 1 according as $n$ is even or odd. Thus $d_{1}=d_{1}$ and we get in particular

$$
\left(2^{t}-1, n\right)=\left(2^{t}+1, n\right)=\left(2^{2 t}-1, n\right)=1,
$$

for all positive odd integers $t$.

Now let $1 \neq p$ be a prime divisor of $m$. If ind, $2 \neq 0(\bmod 4)$, there is an odd or twice-an-odd integer $s$ such that $p \mid 2^{2}-1$ and so $p \mid\left(2^{2}-1, n\right)$ which is contrary to (14). Hence ind $2 \equiv 0(\bmod 4)$. This completes the proof of the theorem.

Remark. If $r=0$ or 1 , then it can be independently proved that $P(G) \subset \Lambda(G)$ if and only if the least positive integer $s$, satisfying $2^{s} \equiv-1$ $(\bmod m)$, is odd and $\Lambda(G) \subset P(G)$ if and only if ind $m 2$ is odd. Further, the proper inclusion of commutation semigroups does not hold if $r>1$.

\section{PART II}

\section{Commutation semigroups of nilpotent groups}

Let $G$ be a nilpotent group. For $l \geqq 1$, let $P_{l}=P_{l}(G)\left(\Lambda_{l}=\Lambda_{l}(G)\right)$ denote the set of all elements of $P(G)(\Lambda(G))$ which can be expressed as a product of $l$ single elements of $P(G)(\Lambda(G))$. Thus if $G$ is nilpotent of class $n$, then $P_{n}=\Lambda_{n}=\{0\}$.

Let $G$ be nilpotent of class at most 4. Since $\rho(a)=\rho(b)$ and $\lambda\left(a^{-1}\right)=$ $\lambda\left(b^{-1}\right)$ are both equivalent to $a b^{-1} \in Z(G)$, the center of $G$, there is a unique one-to-one mapping of $P_{1}$ onto $\Lambda_{1}$ which maps $\rho(a)$ to $\lambda\left(a^{-1}\right)$. Thus we have,

$$
\left|P_{1}\right|=\left|\Lambda_{1}\right| \text {. }
$$

Further since $[g, a, b]=[b,[a, g]]^{[g, a]}=[b,[a, g]]$ for all $g \in G$, we have $\rho(a) \rho(b)=\lambda(a) \lambda(b)$ which gives

$$
P_{2}=\Lambda_{2} \text {. }
$$

Also $[g, a, b, c]=\left[c^{-1},\left[b^{-1},\left[a^{-1}, g\right]\right]\right]$ for all $g \in G$ implies that $\rho(a) \rho(b) \rho(c)=$ $\lambda\left(a^{-1}\right) \lambda\left(b^{-1}\right) \lambda\left(c^{-1}\right)$ which gives

$$
P_{3}=\Lambda_{3}
$$

If $\rho(a)=\rho(b) \rho(c)$, then

$$
\begin{aligned}
{[g, b, c]=[g, a] } & =\left[a^{-1}, g\right]\left[a^{-1}, g, a\right] \\
& =\left[a^{-1}, g\right]\left[a^{-1}, g, b, c\right]=\left[a^{-1}, g\right][g, a, b, c]=\left[a^{-1}, g\right],
\end{aligned}
$$

so that $\rho(a)=\lambda\left(a^{-1}\right)=\rho(b) \rho(c)$ which gives by (2) that 


$$
P_{1} \cap P_{2}=\Lambda_{1} \cap \Lambda_{2} \text {. }
$$

If $\rho(a)=\rho(b) \rho(c) \rho(d)$, then $[g, b, c, d]=[g, a]=\left[a^{-1}, g\right]\left[a^{-1}, g, a\right]=$ $\left[a^{-1}, g\right]$, so that $\rho(a)=\lambda\left(a^{-1}\right)=\rho(b) \rho(c) \rho(d)=\lambda\left(b^{-1}\right) \lambda\left(c^{-1}\right) \lambda\left(d^{-1}\right)$ which gives by (3) that

$$
P_{1} \cap P_{3}=\Lambda_{1} \cap \Lambda_{3} \text {. }
$$

Also from (2) and (3) we get

$$
P_{2} \cap P_{3}=\Lambda_{2} \cap \Lambda_{3} \text {. }
$$

Thus it follows from $(1)-(6)$, that

$$
|P|=|\Lambda| \text {. }
$$

In particular if $G$ is nilpotent of class 2 then $\rho(a)=\lambda\left(a^{-1}\right)$ and so $P=\Lambda$. If $G$ is nilpotent of class 3 , then $\rho(a) \rho(b)=\rho\left(a^{-1}\right) \rho\left(b^{-1}\right)=\lambda\left(a^{-1}\right) \lambda\left(b^{-1}\right)=$ $\lambda(a) \lambda(b)$ and it follows from above that
(i) $\rho(a)=\rho(b)$
if and only if $\lambda\left(a^{-1}\right)=\lambda\left(b^{-1}\right)$
(ii) $\rho(a)=\rho(b) \rho(c)$
if and only if $\lambda\left(a^{-1}\right)=\lambda\left(b^{-1}\right) \lambda\left(c^{-1}\right)$
(iii) $\rho(a) \rho(b)=\rho(c) \rho(d)$ if and only if $\lambda\left(a^{-1}\right) \lambda\left(b^{-1}\right)=\lambda\left(c^{-1}\right) \lambda\left(d^{-1}\right)$.

Thus there exists an isomorphism of $P$ onto $A$ mapping $\rho(a)$ to $\lambda\left(a^{-1}\right)$ for all $a \in G$. Thus we have proved the following theorem:

THEOREM 2. Let $G$ be a nilpotent group. Then (i) $P(G)=\Lambda(G)$ if $G$ has class 2; (ii) $P(G) \cong \Lambda(G)$ if $G$ has class 3 and (iii) $|P(G)|=|\Lambda(G)|$ if $G$ has class 4.

Next we prove the following theorem:

THEOREM 3. If $G$ is a nilpotent group of class 5 , then $P(G)$ and $\Lambda(G)$ are not in general isomorphic.

Proof. To prove this theorem we construct a group $G$ of class precisely 5 and later we shall show that $P ¥ \Lambda$.

First construct,

$$
A=\operatorname{gp}\left\{x_{1}, x_{2}, x_{3}, x_{4}\right\},
$$

as an elementary abelian group of order $5^{4}$. We then extend $A$ by adjoining an element $a$ with relations,

$$
a^{5}=1, x_{1}^{a}=x_{1} x_{2}, x_{2}^{a}=x_{2} x_{3}, x_{3}^{a}=x_{3} x_{4}, x_{4}^{a}=x_{4} .
$$

It can be checked that $a$ induces an automorphism of order 5 in $A$, so that $B=\operatorname{gp}\{A, a\}$ is of order $5^{5}$ and is a splitting extension of $A$ by gp $\{a\}$. In the same way we extend $B$ by adjoining an element $b$ with relations,

$$
b^{b}=1, x_{1}^{b}=x_{1} x_{3}, x_{2}^{b}=x_{2} x_{4}, x_{3}^{b}=x_{3}, x_{4}^{b}=x_{4}, a^{b}=a .
$$

As before $C=\operatorname{gp}\{B, b\}$ is of order $5^{6}$ and is a splitting extension of $B$ by gp $\{b\}$. Finally we extend $C$ by adjoining an element $c$, with relations, 
(11) $c^{5}=1, x_{1}^{c}=x_{1}, x_{2}^{c}=x_{2}, x_{3}^{c}=x_{3}, x_{4}^{c}=x_{4}, a^{e}=a x_{1}^{-1}, b^{c}=b x_{2}^{-1}$.

It can be checked that $G=\operatorname{gp}\{C, c\}$ is of order $5^{7}$ and is a splitting extension of $C$ by gp $\{c\}$.

$G$ is a metabe.ian group and can be regarded as generated by the elements $a, b$ and $c$ with the following commutator relations:

$$
\begin{array}{lll}
{[c, a]=x_{1},} & {[c, b]=x_{2},} & {[a, b]=1,} \\
{[c, a, a]=x_{2},} & {[c, a, b]=x_{3},} & {[c, a, c]=1,} \\
{[c, b, a]=x_{3},} & {[c, b, b]=x_{4},} & {[c, b, c]=1,} \\
{[c, a, a, a]=x_{3},} & {[c, a, a, a, a]=x_{4},} & {[c, a, b, b]=1 .}
\end{array}
$$

Any element $g \in G$ can be written as:

$$
g=a^{u} b^{v} c^{w} z \text { where } u, v, w=0,1,2,3,4
$$

and

$$
z=x_{1}^{i} x_{2}^{j} x_{3}^{k} x_{4}^{l} \text { where } i, j, k, l=0,1,2,3,4 .
$$

In what follows we shall prove that $P(G) \cong \Lambda(G)$.

From relations (12), it can be verified that in $P(G), \rho(a) \rho(a)=\rho(b)$. We prove the following,

LEMMA 1. In $P(G)$,

$$
\rho\left(a c^{i} x_{1}^{j} x_{2}^{k}\right) \rho(a)=\rho\left(b x_{1}^{i} x_{2}^{j} x_{3}^{k}\right),
$$

where $i, j, k=0,1,2,3,4$.

Proof. Let $g \in G$. Then we have

$$
\begin{aligned}
& g \rho\left(a c^{i} x_{1}^{j} x_{2}^{k}\right) \rho(a) \\
& =\left[g, a c^{i} x_{1}^{j} x_{2}^{k}, a\right] \\
& =\left[a^{u} b^{v} c^{v 0} z, a c^{i} x_{1}^{j} x_{2}^{k}, a\right] \\
& =\left[a^{u} b^{v} c^{v 0} z, c^{i} x_{1}^{j} x_{2}^{k}, a\right]\left[a^{u} b^{v} c^{v 0} z, a, a\right] \\
& =\left[a^{u} b^{v}, c^{i} x_{1}^{j} x_{2}^{k}, a\right]\left[c^{* 0} z, b\right] \\
& =\left[a^{u} b^{v}, c^{i}, a\right]\left[a^{u} b^{v}, x_{1}^{j}, a\right]\left[a^{u} b^{v}, x_{2}^{x}, a\right]\left[c^{v 0} z, b\right] \\
& =\left[c^{i}, a^{u} b^{v}, a\right]^{-1}\left[x_{1}^{j}, a^{u} b^{v}, a\right]^{-1}\left[x_{2}^{k}, a^{u} b^{v}, a\right]^{-1}\left[c^{10} z, b\right] \\
& =\left[c^{i}, a, a^{u} b^{\mu}\right]^{-1}\left[x_{1}^{j}, a, a^{u} b^{v}\right]^{-1}\left[x_{2}^{k}, a, a^{u} b^{v}\right]^{-1}\left[c^{* 0} z, b\right] \\
& =\left[[c, a]^{i}, a^{u} b^{v}\right]^{-1}\left[\left[x_{1}, a\right]^{j}, a^{u} b^{v}\right]^{-1}\left[\left[x_{2}, a\right]^{k}, a^{\mu} b^{v}\right]^{-1}\left[c^{*} z, b\right] \\
& =\left[x_{1}^{i}, a^{*} b^{v}\right]^{-1}\left[x_{2}^{j}, a^{w} b^{v}\right]^{-1}\left[x_{3}^{k}, a^{*} b^{v}\right]^{-1}\left[c^{v} z, b\right] \\
& =\left[x_{1}^{j} x_{2}^{j} x_{3}^{k}, a^{*} b^{v}\right]^{-1}\left[c^{*} z, b\right] \\
& =\left[a^{u} b^{0} c^{10} z, x_{1}^{4} x_{2}^{j} x_{3}^{k}\right]\left[a^{u} b^{0} c^{10} z, b\right] \\
& =\left[a^{\mu} b^{*} c^{100} z, b x_{1}^{i} x_{2}^{j} x_{3}^{k}\right] \\
& =g \rho\left(b x_{1}^{i} x_{2}^{v} x_{3}^{k}\right) \text {. }
\end{aligned}
$$


The proofs of the following lemmas are omitted since they are long and computational:

LEMMA 2. $\quad \rho\left(a b^{2} c^{\tau} x_{1}^{s} x_{2}^{t}\right) \rho\left(a^{2}\right)=\rho\left(b^{2} x_{1}^{4} x_{2}^{4} x_{3}^{k}\right)$

where $i, j, k=0,1,2,3,4$; and $r=3 i(\bmod 5), s=(3 j-4 i)(\bmod 5)$, $t=(3 k-4 j+2 i)(\bmod 5)$.

LEMMA 3. $\quad \rho\left(a^{2} b c^{r} x_{1}^{s} x_{2}^{t}\right) \rho\left(a^{4}\right)=\rho\left(b^{3} x_{1}^{i} x_{2}^{j} x_{3}^{k}\right)$

where $i, j, k=0,1,2,3,4$; and $r=4 i(\bmod 5), s=(4 j-i)(\bmod 5)$, $t=(4 k-j)(\bmod 5)$.

$$
\text { LEMMA 4. } \quad \rho\left(a^{2} b^{3} c^{4} x_{1}^{4} x_{2}^{t}\right) \rho\left(a^{2}\right)=\rho\left(b^{4} x_{1}^{i} x_{2}^{j} x_{3}^{k}\right)
$$

where $i, j, k=0,1,2,3,4$; and $r, s, t$ are as in Lemma 2 .

We now prove the following,

LEMMa 5. In $\Lambda(G), \lambda\left(b^{2}\right)$ does not belong to $\Lambda_{2}$.

Proof. Let $h_{1}=a^{i} b^{j} c^{k} z_{1}$ and $h_{2}=a^{i} b^{m} c^{n} z_{2}$ where $i, j, k, l, m, n=$ $0,1,2,3,4$ and $z_{1}, z_{2} \in G^{\prime}$. Suppose that $\lambda\left(b^{2}\right)=\lambda\left(h_{1}\right) \lambda\left(h_{2}\right)$, then $\left[b^{2}, g\right]=\left[h_{2},\left[h_{1}, g\right]\right]$ for all $g \in G$, i.e. $\left[g, b^{2}\right]^{-1}=\left[g, h_{1}, h_{2}\right]$ since $G^{\prime}$ is abelian. In particular we have in turn

$$
\begin{aligned}
& {\left[c, h_{1}, h_{2}\right]=\left[c, b^{2}\right]^{-1} ;} \\
& {\left[c, a^{i} b^{1} c^{k} z_{1}, a^{l} b^{m} c^{n} z_{2}\right]=\left[c, b^{2}\right]^{-1} ;} \\
& {\left[c, a^{i} b^{j}, a^{l} b^{m}\right]=[c, b]^{-2}[c, b, b]^{-1}} \\
& {\left[c, b^{j}, a^{l} b^{m}\right]\left[c, a^{i}, a^{l} b^{m}\right]\left[c, a^{i}, b^{j}, a^{l} b^{m}\right]=[c, b]^{-2}[c, b, b]^{-1} ;} \\
& {\left[c, b^{j}, b^{m}\right]\left[c, b^{j}, a^{l}\right]\left[c, a^{i}, b^{m}\right]\left[c, a^{i}, a^{l}\right]\left[c, a^{i}, a^{l}, b^{m}\right]\left[c, a^{i}, b^{j}, a^{l}\right]} \\
& \quad=[c, b]^{-2}[c, b, b]^{-1} .
\end{aligned}
$$

Collecting the powers of $[c, a, a],[c, a, a, a]$ and $[c, a, a, a, a]$ from the above equation, we get

$$
\begin{aligned}
i l+2 \equiv 0(\bmod 5) & \\
2 i m+2 j l+i l(i+l-2) \equiv 0(\bmod 5) & \\
\frac{i l(l-1)(l-2)}{6} & +\frac{l i(i-1)(i-2)}{6} \\
& +\frac{i(i-1)}{2} \cdot \frac{l(l-1)}{2}+i j l+i l m+j m+\frac{\operatorname{mi}(i-1)}{2} \\
& +\frac{j l(l-1)}{2}+1 \equiv 0(\bmod 5) .
\end{aligned}
$$

From (14) we have, either $i l=3$ or $i l=8$. 
When $i l=3$, let $i=1$ and $l=3$. Then from (15) we have, $m+3 j+3 \equiv 0$ $(\bmod 5)$; which gives $m \equiv-(3 j+3)(\bmod 5)$. Also from $(16)$ we have, $m j+j+3 m+2 \equiv 0(\bmod 5)$; so that $3 j^{2}+j+7 \equiv 0(\bmod 5)$. But this is not solvable for any integral value of $j$ (we arrive at similar conclusion by choosing $i=3$ and $l=1$ ).

When $i l=8$, let $i=2$ and $l=4$. Then from (15) we have, $m+2 j+3 \equiv 0$ $(\bmod 5) ;$ which gives $m \equiv-(2 j+3)(\bmod 5)$. Also from $(16)$ we have, $m j+4 m+4 j \equiv 0(\bmod 5)$; so that $j^{2}+j+1 \equiv 0(\bmod 5)$. But this is again not solvable for any integral value of $j$ (we arrive at similar conclusion by choosing $i=4$ and $l=2$ ). This completes the proof of lemma 5 .

LEMMA 6. In $\Lambda(G), \lambda\left(b^{2}\right)$ is prime.

Proof. By lemma 5, it is sufficient to show that $\lambda\left(b^{2}\right)$ does not belong to $\Lambda_{3}, \Lambda_{4}$ or $\Lambda_{5}$.

But, for $i=3,4,5, \lambda\left(b^{2}\right) \in \Lambda_{i}$ gives in turn $\lambda\left(b^{2}\right) \lambda(a) \lambda(a)=0$; $\rho\left(b^{2}\right) \rho(a) \rho(a)=0 ;\left[c, b^{2}, a, a\right]=1 ;[c, b, a, a]=1$, which gives the required contradiction.

We can now complete the proof of Theorem 3. Let $\bar{P}$ denote the set of all prime elements $\alpha \in P(G)$ such that $\alpha^{2} \neq 0, \alpha^{3}=0, \alpha P_{3}(G)=0$; and let $\bar{\Lambda}$ be the corresponding set of all prime elements $\beta \in \Lambda(G)$ such that $\beta^{2} \neq 0, \beta^{3}=0, \beta \Lambda_{3}(G)=0$.

If $\alpha=\rho\left(a^{i} b^{j} c^{k} z\right) \in \bar{P}$, then $\alpha P_{3}=0$ implies in particular that $\left[c, a^{i} b^{j} c^{k} z, a, a, a\right]=1$ and $\left[a, a^{i} b^{j} c^{k} z, a, a, a\right]=1$ which give respectively $[c, a, a, a, a]^{i}=1$ and $[c, a, a, a, a]^{k}=1$ so that we have $i \equiv 0(\bmod 5)$ and $k \equiv 0(\bmod 5)$. Thus $\alpha=\rho\left(b^{j} z\right)$ which by lemmas $1,2,3$ and 4 implies that $\alpha$ is not prime. Hence $\bar{P}=\emptyset$, the empty set.

On the other hand, by lemma $6, \lambda\left(b^{2}\right)$ is prime and $\lambda^{2}\left(b^{2}\right) \neq 0$, $\lambda^{3}\left(b^{2}\right)=0, \lambda\left(b^{2}\right) \Lambda_{3}(G)=0$ so that $\bar{\Lambda} \neq \emptyset$. Since under any isomorphism of $P$ onto $\Lambda, \bar{P}$ maps onto $\bar{\Lambda}$, we have that $P(G) \neq \Lambda(G)$. This completes the proof.

Finally for $n>5$ we prove the following theorem,

THEOREM 4. For each integer $n$ greater than 5 , there exists a nilpotent group (S) of class $n$ such that $P(\mathbb{S}) \neq \Lambda$ (अ) .

Proof. Let $G$ be the group as constructed in theorem 3 , and let $H$ be the dihedral group of order $2^{n+1}$, given as,

$$
H=\operatorname{gp}\left\{d, e / d^{2^{n}}=1=e^{2}, e d e=d^{-1}\right\} .
$$

Let $\mathbb{B}=G \times H$ be the direct product of $G$ and $H$, then we proceed to show that $\mathfrak{G}$ is the required group.

Since $H$ is of class $n(>5)$, it follows that $\&$ is of class $n$. Every element 
of $\&$ can be uniquely written as $a^{i} b^{j} c^{k} z e^{\varepsilon} d^{l}$ where $i, j, k=0,1,2,3,4$; $\varepsilon=0,1 ; l=1,2, \cdots, 2^{n}$ and $z \in G^{\prime}$.

Let $P(\mathcal{B S})$ denote the set of all prime elements $\alpha \in P(\mathfrak{S})$ such that $\alpha^{2} \neq 0, \alpha^{3}=0, \alpha P_{3}(\mathbb{S})=0$; and let $\bar{\Lambda}(\mathbb{S})$ be the corresponding set of all prime elements $\beta \in \Lambda$ (B) such that $\beta^{2} \neq 0, \beta^{3}=0, \beta \Lambda_{3}(\mathcal{S})=0$.

If $\alpha=\rho\left(a^{i} b^{j} c^{k} z e^{\varepsilon} d^{l}\right) \in \bar{P}(\mathcal{S})$, by using $\alpha P_{\mathbf{3}}(\mathfrak{S})=0$ we get, as in theorem 3 , that $i=0, k=0$; so that $\alpha=\rho\left(b^{j} z e^{\varepsilon} d^{l}\right)$. If $\varepsilon=1$, then $\alpha^{3}=0$ gives in particular $1=\left[d, b^{j} z e d^{l}, b^{j} z e d^{l}, b^{j} z e d^{l}\right]=[d, e, e, e]=d^{-2^{3}}$ which is a contradiction (since $n>5$ ). Thus $\varepsilon=0$. Further $\alpha P_{3}(\mathcal{S})=0$ gives in particular $1=\left[e, b^{j} z d^{l}, e, e, e\right]=[e, d, e, e, e]^{l}=[d, e, e, e, e]^{-l}=d^{-2^{\iota} l}$, which gives that $l=0$ or $l= \pm 2^{n-4}$. Thus $\alpha=\rho\left(b^{j} z\right)$ or $\alpha=\rho\left(b^{j} z d^{ \pm 2^{n-4}}\right)$. But by lemmas $1,2,3$ and $4, \alpha \neq \rho\left(b^{j} z\right)$; therefore $\alpha=\rho\left(b^{j} z d^{ \pm 2^{n-4}}\right)$. Further, since $\rho\left(d \pm 2^{n-4}\right)=\rho\left(d^{\mp 2^{n-8}}\right) \rho(e)$, if $\rho\left(b^{j} z\right)=\rho\left(g_{1}\right) \rho\left(g_{2}\right)$ then it can be easily seen that $\rho\left(b^{s} z d^{ \pm 2^{n-4}}\right)=\rho\left(g_{1} d^{\mp 2^{n-8}}\right) \rho\left(g_{2} e\right)$ and hence $P(\mathscr{S})=\emptyset$.

On the other hand, from theorem 3 , we have $\lambda\left(b^{2}\right) \in \bar{\Lambda}(G)$ and hence $\lambda\left(b^{2}\right) \in \bar{\Lambda}(\mathbb{S})$ so that $\bar{\Lambda}(\mathbb{B S}) \neq \emptyset$. Thus by the argument used in the theorem 3, $P(\mathfrak{H}) ¥ \Lambda(\mathfrak{S})$.

Department of Mathematics

Institute of Advanced Studies

The Australian National University

Canberra 